\title{
ON THE LEBESGUE DECOMPOSITION OF POSITIVE LINEAR FUNCTIONALS
}

\author{
ZSOLT SZŨCS \\ (Communicated by Marius Junge)
}

\begin{abstract}
In this paper we will prove that the Lebesgue decomposition of positive linear functionals on Banach *-algebras corresponds with the Lebesgue decomposition of the nonnegative forms derived from the functionals.
\end{abstract}

S. Hassi, Z. Sebestyén and H. de Snoo recently proved a general Lebesgue type decomposition for forms (i.e. semi-inner products) on complex linear spaces [3]; namely, a form is the sum of its regular and singular part with respect to another form. A similar result on Banach *-algebras for positive functionals has been shown by S. Gudder [2. A natural question arises: if we apply both decompositions for a pair of positive functionals, is there any connection between the two results? The goal of this paper is to answer this question. Since in 3 only forms appear, if one applies the decomposition in 3] to a pair of forms associated with positive functionals, one gets a form decomposition. However, it is not clear that the regular and singular parts in this decomposition are still associated with some positive functionals on $\mathscr{A}$. But it will turn out that if we apply the form decomposition in [3] to the forms associated with positive functionals, then we get Gudder's result, namely the decomposition for positive functionals in [2].

Notation. If $\mathfrak{t}$ is a form on a complex linear space $\mathcal{D}$, then for $x \in \mathcal{D}, \mathfrak{t}[x]:=\mathfrak{t}(x, x)$, i.e. the quadratic form generated by $\mathfrak{t}$. The kernel of $\mathfrak{t}$ will be denoted by ker $\mathfrak{t}$; i.e., ker $\mathfrak{t}:=\{x \in \mathcal{D} \mid \mathfrak{t}[x]=0\}$. If $\mathfrak{w}$ is another form on $\mathcal{D}$, then $\mathfrak{w} \leq \mathfrak{t}$ means that $\mathfrak{w}[x] \leq \mathfrak{t}[x]$ holds for all $x \in \mathcal{D}$.

If $\mathscr{A}$ is a *algebra and $w$ is a positive linear functional on $\mathscr{A}$ (i.e. $w\left(a^{*} a\right) \geq$ $0 \forall a \in \mathscr{A}$ ), then $\widetilde{w}$ stands for the semi-inner product generated by $w$; namely, $\widetilde{w}(a, b)=w\left(b^{*} a\right)$ for all $a, b \in \mathscr{A}$. For positive functionals $v$ and $w, w \leq v$ denotes the natural ordering, i.e. $\widetilde{w} \leq \widetilde{v}$. Denote by $\left(\mathcal{H}_{w},\langle\cdot, \cdot\rangle_{w}\right)$ the associated Hilbert space and semi-inner product from the well-known Gelfand-Naimark-Segal construction (GNS). If $\mathscr{A}$ is a Banach *-algebra, then $r(a)$ stands for the spectral radius of the element $a \in \mathscr{A}$.

For *-algebras, positive functionals and the GNS-construction, see [1, [4, [5], [6], 7].

Let us begin with definitions. The following is from [3]:

Definition 1. Let $\mathcal{D}$ be a complex linear space and let $\mathfrak{t}, \mathfrak{w}$ be forms on $\mathcal{D}$.

Received by the editors September 17, 2010 and, in revised form, July 8, 2011.

2010 Mathematics Subject Classification. Primary 46L51; Secondary 46C50, 46L30.

Key words and phrases. Nonnegative forms, positive functionals, Lebesgue type decomposition, singularity. 
(a) $\mathfrak{w}$ is $\mathfrak{t}$-absolutely continuous (or $\mathfrak{w}$ is closable with respect to $\mathfrak{t}$ ) if for any sequence $\left(x_{n}\right)_{n \in \mathbb{N}}$ in $\mathcal{D}$ the property

$$
\left(\lim _{n \rightarrow+\infty} \mathfrak{t}\left[x_{n}\right]=0\right) \wedge\left(\lim _{n, m \rightarrow+\infty} \mathfrak{w}\left[x_{n}-x_{m}\right]=0\right)
$$

implies that $\lim _{n \rightarrow+\infty} \mathfrak{w}\left[x_{n}\right]=0$.

(b) $\mathfrak{w}$ and $\mathfrak{t}$ are singular if for any form $\mathfrak{p}$ the property

$$
(\mathfrak{p} \leq \mathfrak{w}) \wedge(\mathfrak{p} \leq \mathfrak{t})
$$

implies that $\mathfrak{p}=0$.

The next definition is from article [2]:

Definition 2. Let $\mathscr{A}$ be a ${ }^{*}$-algebra and let $v, w$ be positive functionals on $\mathscr{A}$.

(a) $w$ is $v$-absolutely continuous (or $w$ is absolutely continuous with respect to $v)$ if for any sequence $\left(a_{n}\right)_{n \in \mathbb{N}}$ in $\mathscr{A}$ the property

$$
\left(\lim _{n \rightarrow+\infty} \widetilde{v}\left[a_{n}\right]=0\right) \wedge\left(\lim _{n, m \rightarrow+\infty} \widetilde{w}\left[a_{n}-a_{m}\right]=0\right)
$$

implies that $\lim _{n \rightarrow+\infty} \widetilde{w}\left[a_{n}\right]=0$.

(b) $w$ is $v$-semisingular if there exists a sequence $\left(a_{n}\right)_{n \in \mathbb{N}}$ in $\mathscr{A}$ with the following property:

(1) $\left(\lim _{n \rightarrow+\infty} \widetilde{v}\left[a_{n}\right]=\lim _{n, m \rightarrow+\infty} \widetilde{w}\left[a_{n}-a_{m}\right]=0\right) \wedge \lim _{n \rightarrow+\infty} w\left(a_{n}^{*} a\right)=w(a)(a \in \mathscr{A})$.

Remark 1. In [2] the functionals possessing the property (a) were called strongly $v$-absolutely continuous functionals.

The property $\left(\lim _{n \rightarrow+\infty} \widetilde{v}\left[a_{n}\right]=0\right) \wedge\left(\lim _{n, m \rightarrow+\infty} \widetilde{w}\left[a_{n}-a_{m}\right]=0\right)$ means that $\left(a_{n}+\operatorname{ker} \widetilde{v}\right)_{n \in \mathbb{N}}$ is a zero-sequence in the space $\mathcal{H}_{v}$; meanwhile $\left(a_{n}+\operatorname{ker} \widetilde{w}\right)_{n \in \mathbb{N}}$ is Cauchy in $\mathcal{H}_{w}$. We also note that Definition 1 (a) and Definition 2 (a) are just the same for a pair of forms associated with positive functionals.

Before we come to the Lebesgue decompositions, we examine the definition of semisingularity. Our first result shows that when the algebra has unit element then we can simplify the definition.

Proposition 1. Let $\mathscr{A}$ be $a^{*}$-algebra with unit and let $v, w$ be positive functionals on $\mathscr{A}$. Then

(a) (1) holds if and only if $\widetilde{v}\left[a_{n}\right] \rightarrow 0$ and $\widetilde{w}\left[\mathbf{1}-a_{n}\right] \rightarrow 0$, i.e. $a_{n}+\operatorname{ker} \widetilde{v} \rightarrow$ $0+\operatorname{ker} \widetilde{v}$ and $a_{n}+\operatorname{ker} \widetilde{w} \rightarrow \mathbf{1}+\operatorname{ker} \widetilde{w}(n \rightarrow+\infty)$.

(b) $w$ is $v$-semisingular if and only if $v$ is $w$-semisingular.

Proof. (a): First assume that (10) holds. We have to prove that $\widetilde{w}\left[\mathbf{1}-a_{n}\right] \rightarrow 0$. The sequence $\left(a_{n}+\operatorname{ker} \widetilde{w}\right)_{n \in \mathbb{N}}$ is Cauchy in the Hilbert space $\mathcal{H}_{w}$; therefore it converges to a vector $\xi \in \mathcal{H}_{w}$. Hence for every $a \in \mathscr{A}$ we have that

$$
\left\langle a+\operatorname{ker} \widetilde{w}, a_{n}+\operatorname{ker} \widetilde{w}\right\rangle_{w} \rightarrow\langle a+\operatorname{ker} \widetilde{w}, \xi\rangle_{w} .
$$

At the same time the second property of $v$-semisingularity implies that

$$
\left\langle a+\operatorname{ker} \widetilde{w}, a_{n}+\operatorname{ker} \widetilde{w}\right\rangle_{w}=w\left(a_{n}^{*} a\right) \rightarrow w(a)=\langle a+\operatorname{ker} \widetilde{w}, \mathbf{1}+\operatorname{ker} \widetilde{w}\rangle_{w} ;
$$

thus for every $a \in \mathscr{A}$ we obtain

$$
\langle a+\operatorname{ker} \widetilde{w}, \xi-(\mathbf{1}+\operatorname{ker} \widetilde{w})\rangle_{w}=0 .
$$


This means that the vector $\xi-(\mathbf{1}+\operatorname{ker} \widetilde{w})$ is orthogonal to the elements of the dense linear subspace $\mathscr{A} / \operatorname{ker} \widetilde{w}$; hence $\xi-(\mathbf{1}+\operatorname{ker} \widetilde{w})=0$, that is, $\xi=\mathbf{1}+\operatorname{ker} \widetilde{w}$. Consequently $\widetilde{w}\left[\mathbf{1}-a_{n}\right] \rightarrow 0$.

Conversely assume that $\widetilde{v}\left[a_{n}\right] \rightarrow 0$ and $\widetilde{w}\left[\mathbf{1}-a_{n}\right] \rightarrow 0$, and fix an arbitrary $a \in \mathscr{A}$. By the Cauchy-Schwarz inequality we have

$$
\begin{aligned}
& \left|w(a)-w\left(a_{n}^{*} a\right)\right|^{2}=\left|w\left(\left(\mathbf{1}-a_{n}\right)^{*} a\right)\right|^{2} \\
& \leq w\left(\left(\mathbf{1}-a_{n}\right)^{*}\left(\mathbf{1}-a_{n}\right)\right) w\left(a^{*} a\right)=\widetilde{w}\left[\mathbf{1}-a_{n}\right] \widetilde{w}[a] \rightarrow 0
\end{aligned}
$$

hence $w\left(a_{n}^{*} a\right) \rightarrow w(a)$, i.e. (11) holds.

(b): It is enough to prove that if $w$ is $v$-semisingular, then $v$ is $w$-semisingular. By (a) there exists a sequence $\left(a_{n}\right)_{n \in \mathbb{N}}$ such that $\widetilde{v}\left[a_{n}\right] \rightarrow 0$ and $\widetilde{w}\left[\mathbf{1}-a_{n}\right] \rightarrow 0$. Then for the sequence $\left(b_{n}\right)_{n \in \mathbb{N}}=\left(\mathbf{1}-a_{n}\right)_{n \in \mathbb{N}}$ we have that

$$
\widetilde{w}\left[b_{n}\right]=\widetilde{w}\left[\mathbf{1}-a_{n}\right] \rightarrow 0, \widetilde{v}\left[\mathbf{1}-b_{n}\right]=\widetilde{v}\left[\mathbf{1}-\left(\mathbf{1}-a_{n}\right)\right]=\widetilde{v}\left[a_{n}\right] \rightarrow 0 ;
$$

thus $v$ is $w$-semisingular according to (a).

Remark 2. By part (b) of the previous proposition we may rightfully say that $v$ and $w$ are singular instead of $w$ is $v$-semisingular or $v$ is $w$-semisingular.

Hereinafter we state the Lebesgue decompositions relative to forms and relative to positive functionals. The first theorem is due to S. Hassi, Z. Sebestyén and H. de Snoo ([3], Theorem 2.11, Theorem 3.9):

Theorem 1 (Lebesgue decomposition of forms). Let $\mathfrak{w}, \mathfrak{t}$ be forms on the complex linear space $\mathcal{D}$. Then $\mathfrak{w}$ admits a decomposition $\mathfrak{w}=\mathfrak{w}_{\text {reg }}+\mathfrak{w}_{\text {sing }}$ to a sum of forms, where $\mathfrak{t}$ and $\mathfrak{w}_{\text {sing }}$ are singular, and $\mathfrak{w}_{\text {reg }}$ is $\mathfrak{t}$-absolutely continuous. The form $\mathfrak{w}_{\text {reg }}$ is the greatest among all of the forms $\mathfrak{p}$ such that $\mathfrak{p} \leq \mathfrak{w}$ and $\mathfrak{p}$ is absolutely continuous with respect to $\mathrm{t}$.

Remark 3 . The forms $\mathfrak{w}_{\text {reg }}$ and $\mathfrak{w}_{\text {sing }}$ are called the regular (absolutely continuous) and singular part of $\mathfrak{w}$, respectively.

The following theorem is due to S. Gudder ([2], Corollary 2 and Corollary 3):

Theorem 2 (Lebesgue decomposition of positive functionals). Let $v$ and $w$ be positive linear functionals on a Banach ${ }^{*}$-algebra $\mathscr{A}$ with unit. Then $w$ admits a decomposition $w=w_{r}+w_{s}$ to a sum of positive functionals, where $v$ and $w_{s}$ are singular, and $w_{r}$ is v-absolutely continuous. Moreover, there exists a sequence $\left(a_{n}\right)_{n \in \mathbb{N}}$ which shows the singularity of $v$ and $w_{s}$, and with the property $\widetilde{w}\left[a_{n}-a_{m}\right] \rightarrow$ 0 .

If we compare the decompositions in Theorems 1 and 2 for the pair of forms associated with positive functionals $v$ and $w$, then by the extremal property of $\mathfrak{w}_{\text {reg }}$ we obtain that $\widetilde{w_{r}} \leq \mathfrak{w}_{\text {reg }}$ holds for the regular parts of $w$. In the proof of Theorem 1 only forms appear, so it is not clear that more can be acquired than $\widetilde{w_{r}} \leq \mathfrak{w}_{\text {reg }}$. The next theorem, our main result, clarifies this problem, and in addition we may conclude that the decompositions are equal.

Theorem 3. Let $\mathscr{A}$ be a Banach ${ }^{*}$-algebra with unit and let $v, w$ be positive functionals on $\mathscr{A}$. Let $w=w_{r}+w_{s}$ be the Lebesgue decomposition of $w$ with respect to $v$ by Theorem 2 and let $\widetilde{w}=\mathfrak{w}_{\text {reg }}+\mathfrak{w}_{\text {sing }}$ be the Lebesgue decomposition of $\widetilde{w}$ with respect to $\widetilde{v}$ by Theorem 1 , Then

$$
\widetilde{w_{r}}=\mathfrak{w}_{\text {reg }}, \widetilde{w_{s}}=\mathfrak{w}_{\text {sing }} .
$$


Proof. It is enough to prove that the equality $\widetilde{w_{r}}[a]=\mathfrak{w}_{r e g}[a]$ holds for all $a \in \mathscr{A}$. Fix an arbitrary $a \in \mathscr{A}$. Since $\widetilde{w_{r}} \leq \mathfrak{w}_{\text {reg }}$ holds, therefore rearranging the equality

$$
\widetilde{w}=\mathfrak{w}_{\text {reg }}+\mathfrak{w}_{\text {sing }}=\widetilde{w_{r}}+\widetilde{w_{s}}
$$

we conclude that

$$
0 \leq \mathfrak{w}_{\text {reg }}-\widetilde{w_{r}}=\widetilde{w_{s}}-\mathfrak{w}_{\text {sing }} \leq \widetilde{w_{s}} .
$$

By Theorem 2 and Proposition 1 there exists a sequence $\left(a_{n}\right)_{n \in \mathbb{N}}$ in $\mathscr{A}$ with the following properties:

$$
\widetilde{v}\left[a_{n}\right] \rightarrow 0, \widetilde{w}\left[a_{n}-a_{m}\right] \rightarrow 0, \widetilde{w_{s}}\left[\mathbf{1}-a_{n}\right] \rightarrow 0 .
$$

In Banach *-algebras it is well-known that for arbitrary $c, d \in \mathscr{A}$ and a positive functional $f$ on $\mathcal{A}$,

$$
\widetilde{f}[c d]=f\left(d^{*} c^{*} c d\right) \leq r\left(c^{*} c\right) f\left(d^{*} d\right)=r\left(c^{*} c\right) \tilde{f}[d]
$$

holds (see 4]); hence applying this estimate along with (3) to the sequence $\left(a a_{n}\right)_{n \in \mathbb{N}}$,

$$
\begin{gathered}
\widetilde{v}\left[a a_{n}\right] \leq r\left(a^{*} a\right) \widetilde{v}\left[a_{n}\right] \rightarrow 0, \\
\widetilde{w}\left[a a_{n}-a a_{m}\right]=\widetilde{w}\left[a\left(a_{n}-a_{m}\right)\right] \leq r\left(a^{*} a\right) \widetilde{w}\left[a_{n}-a_{m}\right] \rightarrow 0,
\end{gathered}
$$

and

$$
\widetilde{w_{s}}\left[a-a a_{n}\right]=\widetilde{w_{s}}\left[a\left(\mathbf{1}-a_{n}\right)\right] \leq r\left(a^{*} a\right) \widetilde{w_{s}}\left[\mathbf{1}-a_{n}\right] \rightarrow 0
$$

follow. Since $\widetilde{w_{r}}[b] \leq \widetilde{w}[b]$ and $\mathfrak{w}_{\text {reg }}[b] \leq \widetilde{w}[b]$ hold for any $b \in \mathscr{A}$, from (5) it follows that

$$
\widetilde{w_{r}}\left[a a_{n}-a a_{m}\right] \rightarrow 0, \mathfrak{w}_{r e g}\left[a a_{n}-a a_{m}\right] \rightarrow 0 .
$$

The forms $\mathfrak{w}_{\text {reg }}$ and $\widetilde{w_{r}}$ are absolutely continuous with respect to the form $\widetilde{v}$; hence from (41) and (7) we conclude that

$$
\mathfrak{w}_{r e g}\left[a a_{n}\right] \rightarrow 0, \widetilde{w_{r}}\left[a a_{n}\right] \rightarrow 0 .
$$

Now for the elements of $\left(a-a a_{n}\right)_{n \in \mathbb{N}}$, (2) implies that

$$
0 \leq \mathfrak{w}_{r e g}\left[a-a a_{n}\right]-\widetilde{w_{r}}\left[a-a a_{n}\right] \leq \widetilde{w_{s}}\left[a-a a_{n}\right] \quad(n \in \mathbb{N})
$$

Tending to infinity with $n$, (6) and (8) yield that

$$
0 \leq \mathfrak{w}_{r e g}[a]-\widetilde{w_{r}}[a] \leq 0,
$$

that is, $\mathfrak{w}_{r e g}[a]=\widetilde{w_{r}}[a]$, and consequently $\widetilde{w_{s}}[a]=\mathfrak{w}_{\text {sing }}[a]$.

As a consequence we may establish an extremal property of the absolutely continuous part $w_{r}$ :

Corollary 1. The positive functional $w_{r}$ is the greatest among all of the positive functionals $f$ such that $f \leq w$ and $f$ is absolutely continuous with respect to $v$.

Proof. Combining the previous theorem and Theorem 1 we conclude that $\widetilde{w_{r}}$ is the greatest form among forms $\mathfrak{f}$ such that $\mathfrak{f} \leq \widetilde{w}$ and $\mathfrak{f}$ is absolutely continuous with respect to $\widetilde{v}$. Specifically, if $\mathfrak{f}$ is derived from a positive functional $f$, then $f \leq w_{r}$. 
Another consequence of Theorem 3 is that the pair of positive functionals $v$ and $w$ are singular if and only if the regular part $w_{r}$ of $w$ with respect to $v$ is identically zero. Furthermore, the definitions of singularity are equivalent for positive functionals; namely, $v$ and $w$ are singular if and only if the forms $\widetilde{v}$ and $\widetilde{w}$ are singular.

Corollary 2. Let $\mathscr{A}$ be a Banach ${ }^{*}$-algebra with unit and let $v, w$ be positive functionals on $\mathscr{A}$. Let $w=w_{r}+w_{s}$ be the Lebesgue decomposition of $w$ with respect to $v$ by Theorem 2. The following statements are equivalent:

(i) $w_{r}=0$.

(ii) For any form $\mathfrak{p}$ on $\mathscr{A}$ the property $(\mathfrak{p} \leq \widetilde{w} \wedge \mathfrak{p} \leq \widetilde{v})$ implies that $\mathfrak{p}=0$.

(iii) There exists a sequence $\left(a_{n}\right)_{n \in \mathbb{N}}$ in $\mathscr{A}$ such that $\widetilde{v}\left[a_{n}\right] \rightarrow 0$ and $\widetilde{w}\left[\mathbf{1}-a_{n}\right] \rightarrow$ 0 .

Proof. (i) $\Leftrightarrow$ (ii): By Theorem 3, (i) holds if and only if $\mathfrak{w}_{r e g}=0$ holds in the form decomposition of $\widetilde{w}$ by Theorem 1 . Hence Corollary 3.11 in [3] shows the equivalence of (i) and (ii).

(i) $\Leftrightarrow$ (iii): If $w_{r}=0$, then $w=w_{s}$ holds. Now (iii) immediately follows, since $w_{s}$ and $v$ are singular.

On the other hand, by $w_{r} \leq w$ and (iii) we have that $\widetilde{w_{r}}\left[\mathbf{1}-a_{n}\right] \rightarrow 0$. But $w_{r}$ is $v$-absolutely continuous and $\widetilde{w_{r}}\left[a_{n}-a_{m}\right] \rightarrow 0$, so $\widetilde{w_{r}}\left[a_{n}\right] \rightarrow 0$, that is, $\widetilde{w_{r}}[\mathbf{1}]=0$. Hence for every $a \in \mathscr{A}$ we obtain that

$$
\left|w_{r}(a)\right|^{2}=\left|w_{r}\left(\mathbf{1}^{*} a\right)\right|^{2}=\left|\left\langle a+\operatorname{ker} \widetilde{w_{r}}, \mathbf{1}+\operatorname{ker} \widetilde{w_{r}}\right\rangle_{w_{r}}\right|^{2} \leq \widetilde{w_{r}}[a] \widetilde{w_{r}}[\mathbf{1}]=0 .
$$

The proof is complete.

\section{ACKNOWLEDGEMENT}

The author is grateful to Professor János Kristóf for several helpful conversations and valuable suggestions.

\section{REFERENCES}

1. J. Dixmier, $C^{*}$-algebras. Translated from the French by Francis Jellett. North-Holland Mathematical Library, Vol. 15, North-Holland Publishing Co., Amsterdam-New York-Oxford, 1977. MR0458185(56:16388)

2. S. P. Gudder, A Radon-Nikodým theorem for *-algebras, Pacific J. Math., Vol. 80, No. 1 (1979), 141-149. MR534703 (80g:46057)

3. S. Hassi, Z. Sebestyén, H. de Snoo, Lebesgue type decompositions for nonnegative forms, Journal of Functional Analysis, Vol. 257, Issue 12 (2009), 3858-3894. MR2557727 (2010k:47042)

4. T. W. Palmer, Banach Algebras and the General Theory of*-Algebras II. Cambridge University Press (2001). MR1819503 (2002e:46002)

5. G. K. Pedersen, $C^{*}$-algebras and their automorphism groups. London Mathematical Society Monographs, 14, Academic Press, Inc. [Hartcourt Brace Jovanovich, Publishers], London-New York, 1979. MR548006 (81e:46037)

6. S. Sakai, $C^{*}$-algebras and $W^{*}$-algebras. Ergebnisse der Mathematik und ihrer Grezengebeite, Band 60, Springer-Verlag, New York-Heidelberg, 1971. MR0442701 (56:1082)

7. Z. Sebestyén, On representability of linear functionals on *-algebras, Period. Math. Hungar. 15 (1984), no. 3, 233-239. MR756188 (86a:46068)

Department of Applied Analysis, Eötvös Loránd University, PÁzmány PÉter sétány 1/C, 1117 Budapest, Hungary

E-mail address: szzsolti@cs.elte.hu 